\title{
Modelling the Effects of Propafenone on Human Atrial Patho-Electrophysiology Associated With hERG-Linked Short QT Syndrome
}

\author{
Dominic G Whittaker ${ }^{1,2}$, Jules C Hancox ${ }^{2,3}$, Henggui Zhang ${ }^{2}$ \\ ${ }^{1}$ University of Leeds, Leeds, UK \\ ${ }^{2}$ University of Manchester, Manchester, UK \\ ${ }^{3}$ University of Bristol, Bristol, UK
}

\begin{abstract}
The N588K mutation to the human Ether-à-go-goRelated Gene (hERG) underlies short QT syndrome variant 1 (SQT1), which is associated with atrial fibrillation (AF). However, mechanisms and management of AF in the context of SQT1 remain poorly understood.

In this study, multi-scale computational modelling was used to investigate pharmacotherapeutic effects of the class Ic drug propafenone for SQT1-mediated human atrial patho-electrophysiology. A Markov chain formulation of rapid delayed rectifier potassium current, $I_{K r}$, describing wild type (WT) and $N 588 K$ mutant currents was incorporated into a recent model of the human atrial action potential (AP), which was integrated into multi-scale tissue models. Effects of multi-channel block by propafenone were simulated on single- and multi-cellular electrophysiology models.

At the single cell level, propafenone prolonged the AP duration under SQT1 (heterozygous N588K) conditions in a dose-dependent manner. In tissue, propafenone prolonged the effective refractory period and excitation wavelength, whilst reducing the conduction velocity. In $2 D$ sheet simulations, propafenone demonstrated efficacy in pharmacological conversion of re-entry. Our findings suggest that propafenone shows efficacy in reversing $A F$ associated with hERG-linked short QT syndrome.
\end{abstract}

\section{Introduction}

The short QT syndrome (SQTS) is characterised primarily by a short QT interval on the ECG, which corresponds to abbreviated ventricular repolarisation. However, symptomatic atrial fibrillation (AF) has also been reported as a common first clinical presentation [1], suggesting that pathophysiological mechanisms leading to shortening of the QT interval also affect the atria, which can increase susceptibility to AF. The N588K mutation to the human Ether-à-go-go-Related gene (hERG), which encodes the $\alpha$ subunit of channels carrying rapid delayed rectifier potassium current, $I_{\mathrm{Kr}}$, underlies SQTS variant 1
(SQT1) [2], and has been associated with a high incidence of AF in affected patients - as high as 50\% [1]. However, effective management of AF in this context remains challenging [3].

The class Ia anti-arrhythmic drug quinidine is typically used as the frontline therapy for QT normalisation in SQT1 [1], and disopyramide has been suggested as a possible alternative [4]. Regarding management of atrial arrhythmias, the class Ic anti-arrhythmic drug propafenone has been reported to be effective at preventing recurrent episodes of paroxysmal $\mathrm{AF}$ mediated by the N588K mutation to hERG, maintaining 2 patients free of arrhythmia recurrence for $>2$ years [5]. However, mechanisms by which propafenone provides beneficial effects in the context of SQT1-mediated atrial proarrhythmia remain unclear, and have not yet been investigated using a multi-scale computational approach. The aim of this study was to assess the efficacy of the class Ic drug propafenone on human atrial pathoelectrophysiology associated with SQT1, using drug binding models with multi-channel pharmacology.

\section{Methods}

\subsection{Model development}

Human atrial electrophysiology was simulated using the recent model of $\mathrm{Ni}$ et al. [6], in which the equations for $I_{\mathrm{Kr}}$ were replaced with previously developed and validated Markov chain formulations of wild type (WT) and N588K mutant $I_{\mathrm{Kr}} / \mathrm{hERG}$ [7]. The resulting model in the WT condition had an action potential (AP) duration at $90 \%$ repolarisation $\left(\mathrm{APD}_{90}\right)$ of $248.8 \mathrm{~ms}$ at $1 \mathrm{~Hz}$. A heterozygous WT-N588K condition (as observed in SQT1 probands), hereinafter referred to as the SQT1 condition, was constructed by considering $I_{\mathrm{Kr}}$ to consist of 50\% WT and 50\% N588K mutant subunits [7], [8].

Using the same approach as detailed for disopyramide and quinidine in our previous study [7], state-dependent models of drug block were developed, incorporating known actions of propafenone on $I_{\mathrm{Kr}} / \mathrm{hERG}$ [9] (including 
reduced potency against N588K-hERG mutant channels [10]) and $I_{\mathrm{Na}}$ [11] (using guarded receptor equations). Propafenone is a multi-channel blocker, and thus exerts secondary effects on other ion channels, which were modelled using a simple pore block based on half maximal inhibitory concentration $\left(\mathrm{IC}_{50}\right)$ values from the literature, summarised in Table 1.

Table 1. A summary of half maximal inhibitory concentration $\left(\mathrm{IC}_{50}\right)$ values extracted from the literature.

\begin{tabular}{lll}
\hline Current & $\mathrm{IC}_{50}(\mu \mathrm{M})$ & Source \\
\hline$I_{\mathrm{CaL}}$ & 1.7 & {$[12]$} \\
$I_{\mathrm{to}}$ & 4.8 & {$[13]$} \\
$I_{\mathrm{K} 1}$ & 16.8 & {$[14]$} \\
$I_{\text {Kur }}$ & 4.4 & {$[15]$} \\
\hline
\end{tabular}

Taking into account plasma protein binding, the most likely unbound concentrations of propafenone have been given as 0.2-0.6 $\mu \mathrm{M}$ [9]. Based on this estimate, the effects of 3 equally-spaced concentrations were investigated in this study $-0.2,0.5$, and $0.8 \mu \mathrm{M}$. Reductions in the model maximum upstroke velocity $\left(\dot{\mathrm{V}}_{\text {max }}\right)$ upon application of propafenone in control conditions were compared with experimental data [16], where close concordance between simulation and experiment can be seen (Figure 1).

\subsection{Tissue simulations}

Propagation of excitation waves in tissue was described using the monodomain equation,

$$
\frac{\partial V}{\partial t}=\nabla(\mathrm{D} \nabla V)-\frac{I_{\text {ion }}}{C_{\mathrm{m}}}
$$

where $V$ is the transmembrane voltage, $\mathbf{D}$ is the global conductivity tensor (set to give a conduction velocity of $\sim 0.7 \mathrm{~m} / \mathrm{s}), I_{\text {ion }}$ is the total ionic current, and $C_{\mathrm{m}}$ is the membrane capacitance. Equation (1) was solved using a finite difference PDE solver based on the explicit forward Euler method and Strang splitting scheme. Effects of the N588K-hERG mutation and its modulation by propafenone on effective refractory period (ERP), conduction velocity (CV), excitation wavelength (WL; given by $\mathrm{WL}=\mathrm{CV} \times \mathrm{ERP}$ ), and spiral wave dynamics were determined using $1 \mathrm{D}$ and 2D atrial tissue models with spatial step, $\Delta x=0.25 \mathrm{~mm}$, as described previously [17], [18]. Re-entry in the 2D sheet was initiated using an $\mathrm{S} 1-\mathrm{S} 2$ cross-shock protocol.

\section{Results}

\subsection{Single cell investigations}

The human atrial $\mathrm{APD}_{90}$ at a pacing rate of $1 \mathrm{~Hz}$ was shortened from $248.8 \mathrm{~ms}$ in the WT condition to $195.4 \mathrm{~ms}$ in SQT1 (WT-N588K) conditions, and ERP was reduced from 273 to $187 \mathrm{~ms}$. Figure 2 shows AP profiles under drug-free SQT1 conditions and upon application of different concentrations of propafenone, with corresponding fractional block of $I_{\mathrm{Kr}}$ and $I_{\mathrm{Na}}$. Propafenone produced only modest prolongations of the APD, with the highest concentration tested $(0.8 \mu \mathrm{M})$ extending the $\mathrm{APD}_{90}$ to $227.4 \mathrm{~ms}$, thus failing to restore it to that of the WT condition. In contrast, propafenone prolonged the ERP effectively, restoring it to $271 \mathrm{~ms}$ at a concentration of $0.8 \mu \mathrm{M}$, close to that of the WT level $(273 \mathrm{~ms})$, due to additional ERP-prolonging effects of $I_{\mathrm{Na}}$ block.

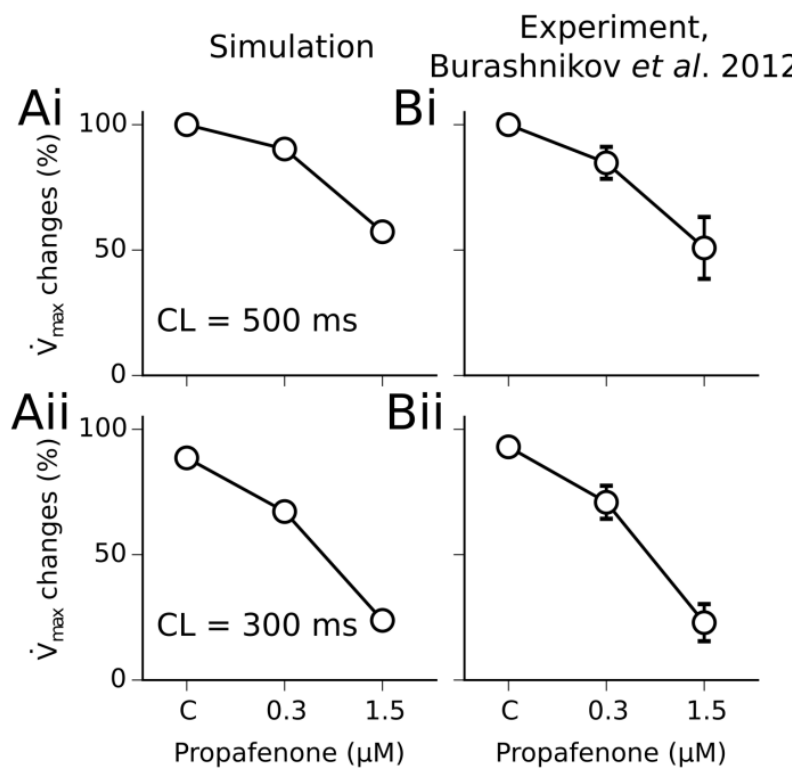

Figure 1. (A) Simulated and (B) experimentally-measured reduction in maximum upstroke velocity $\left(\dot{\mathrm{V}}_{\text {max }}\right)$ by 0.3 and $1.5 \mu \mathrm{M}$ propafenone at a cycle length (CL) of (i) 500 ms and (ii) $300 \mathrm{~ms}$. All $\dot{\mathrm{V}}_{\text {max }}$ reductions are relative to the control (C) value at a CL of $500 \mathrm{~ms}$. Experimental data were recorded from canine atrial myocytes [16].

\subsection{Tissue simulations}

The rate-dependent effects of propafenone on the ERP, $\mathrm{CV}$, and WL in the 1D tissue model are shown in Figure 3. Although the ERP was increased by propafenone in a dose-dependent manner, the degree of prolongation was smaller at fast pacing rates compared to slow pacing rates (reverse use dependence). At very fast pacing rates, the decrease in CV was roughly commensurate with the increase in ERP, and thus the excitation WL did not change. In addition, propafenone promoted beat-to-beat alternans in the model. 


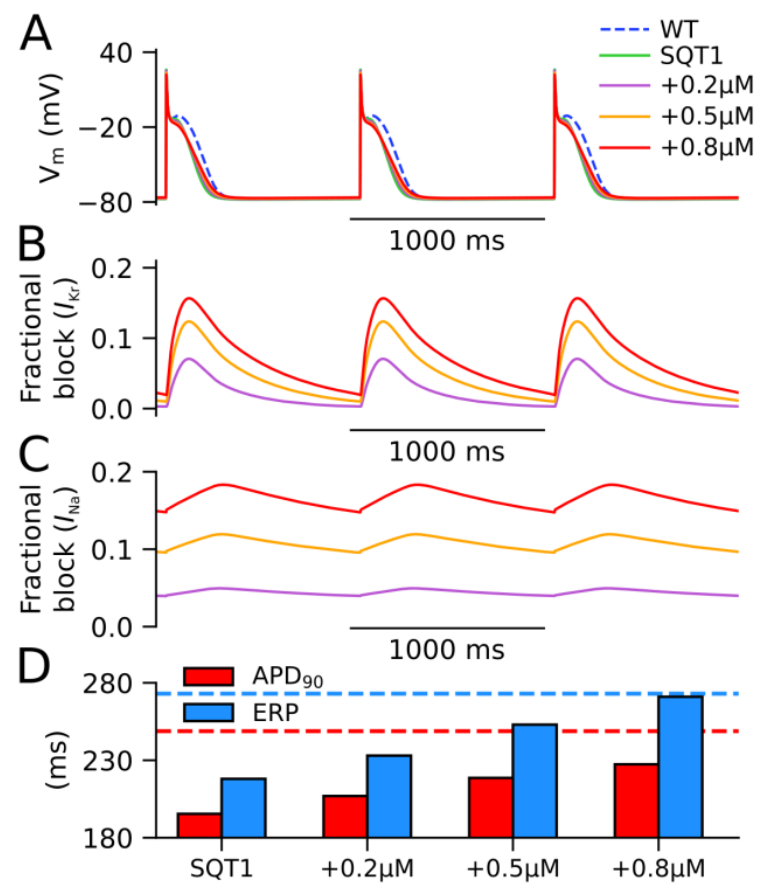

Figure 2. (A) Single human atrial cell APs under WT conditions (blue, dashed line), drug-free SQT1 conditions (green, solid line), and SQT1 + increasing propafenone concentrations (purple, orange, and red solid lines). Corresponding fractional block of (B) $I_{\mathrm{Kr}}$ and (C) $I_{\mathrm{Na}}$, and (D) increase in $\mathrm{APD}_{90}$ (red) and ERP (blue).

In 2D sheets of human atrial tissue using an $\mathrm{S} 1$ pacing interval of $400 \mathrm{~ms}$, an S2 stimulus delivered in the SQT1 condition produced a spiral wave which sustained for the $5.0 \mathrm{~s}$ duration of the simulation, following an epicycloidal trajectory (Figure 4). Application of 0.2 and $0.5 \mu \mathrm{M}$ propafenone resulted in termination of re-entry by increasing the area of meander and inducing secondary waves. Application of $0.8 \mu \mathrm{M}$ propafenone did not terminate re-entry, but did destabilise the re-entrant circuit and increase the area of meander.

\section{Discussion}

In this study, a novel model of the actions of the class Ic drug propafenone was developed and applied to investigate the utility of pharmacotherapy for AF with propafenone in the context of SQT1 using models of human atrial tissue. The heterozygous N588K-hERG mutation shortened the human atrial APD by $53.4 \mathrm{~ms}$, consistent with a previous simulation study [8], as well as the ERP. Propafenone at clinically-relevant conditions produced only modest prolongations of the APD, consistent with experimental results from canine atrial tissue [16], but prolonged ERP effectively in a dosedependent manner due to combined $I_{\mathrm{Kr}}$ and $I_{\mathrm{Na}}$ block. Propafenone also reduced CV dose-dependently, roughly increasing WL at BCL $>400 \mathrm{~ms}$ and not affecting WL at $\mathrm{BCL}<400 \mathrm{~ms}$. Increased ERP and beat-to-beat alternans caused by propafenone promoted re-entry termination in a 2D sheet of atrial tissue, consistent with efficacy of propafenone for AF in SQT1 [5]. As propafenone has been reported to have no prolonging effect on the QT interval in this context [5], however, alternatives such as quinidine may be more suitable for pharmacotherapy [1].
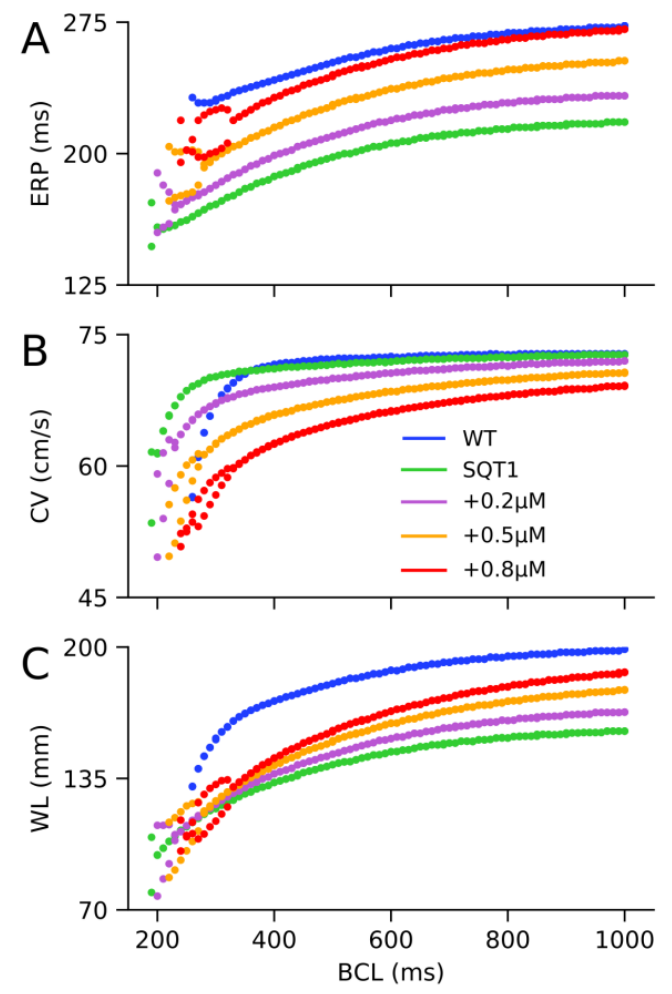

Figure 3. Effects of various concentrations of propafenone (purple, orange, and red for increasing concentrations) on SQT1 mutant (green) atrial tissue, measured using a 1D strand model. The effects of propafenone on the (A) ERP, (B) CV, and (C) WL are shown, and compared with the WT (blue) for reference. For each basic cycle length (BCL), the two final APs were analysed to account for beat-to-beat alternans.

This study further substantiates a causative link between the SQT1-linked N588K-hERG mutation and APD/ERP shortening in human atria which promotes development of AF. Propafenone was shown to increase ERP in a dose-dependent manner in the context of SQT1, which had an anti-arrhythmic effect by increasing tissue excitation WL and promoting re-entry termination.

\section{Acknowledgements}

This work was funded by the British Heart Foundation (FS/14/5/30533 - HZ and JCH) and a Wellcome Trust 
ISSF Fellowship (204825/Z/16/Z - DGW).

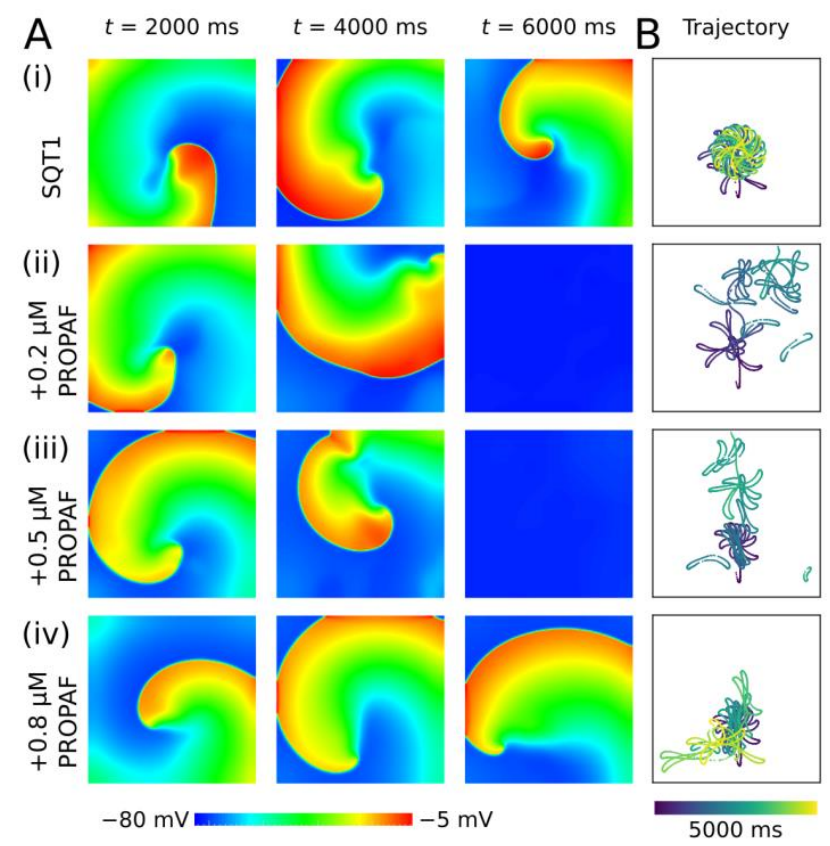

Figure 4. Effects of propafenone (PROPAF) on re-entry in a 2D sheet of SQT1 mutant atrial tissue. (A) Spiral waves under (i) drug-free SQT1 conditions, and upon application of (ii) $0.2 \mu \mathrm{M}$, (iii) $0.5 \mu \mathrm{M}$, and (iv) $0.8 \mu \mathrm{M}$ PROPAF at times $t=2000,4000$, and $6000 \mathrm{~ms}$. (B) Corresponding trajectories over a $5000 \mathrm{~ms}$ period.

\section{References}

[1] D. Hu et al., "The phenotypic spectrum of a mutation hotspot responsible for the short QT syndrome," JACC Clin. Electrophysiol., 2017.

[2] R. Brugada et al., "Sudden death associated with short-QT syndrome linked to mutations in HERG," Circulation, vol. 109, no. 1, pp. 30-35, Jan. 2004.

[3] J. C. Hancox, D. G. Whittaker, C. Du, A. G. Stuart, and H. Zhang, "Emerging therapeutic targets in the short QT syndrome," Expert Opin. Ther. Targets, Apr. 2018.

[4] R. Schimpf, C. Veltmann, C. Giustetto, F. Gaita, M. Borggrefe, and C. Wolpert, "In vivo effects of mutant HERG K+ channel inhibition by disopyramide in patients with a short QT-1 syndrome: A pilot study," $J$. Cardiovasc. Electrophysiol., vol. 18, no. 11, pp. $1157-$ 1160, Nov. 2007.

[5] K. Hong, P. Bjerregaard, I. Gussak, and R. Brugada, "Short QT syndrome and atrial fibrillation caused by mutation in KCNH2," J. Cardiovasc. Electrophysiol., vol. 16, no. 4, pp. 394-396, Apr. 2005.

[6] H. Ni, D. G. Whittaker, W. Wang, W. R. Giles, S. M. Narayan, and H. Zhang, "Synergistic anti-arrhythmic effects in human atria with combined use of sodium blockers and acacetin," Front. Physiol., vol. 8, 2017.

[7] D. G. Whittaker, H. Ni, A. P. Benson, J. C. Hancox, and H. Zhang, "Computational analysis of the mode of action of disopyramide and quinidine on hERG-linked short QT syndrome in human ventricles," Front. Physiol., vol. 8, Oct. 2017.

[8] A. Loewe, M. Wilhelms, F. Fischer, E. P. Scholz, O. Dössel, and G. Seemann, "Arrhythmic potency of human ether-à-go-go-related gene mutations L532P and N588K in a computational model of human atrial myocytes," Europace, vol. 16, no. 3, pp. 435-443, Mar. 2014.

[9] A. A. Paul, H. J. Witchel, and J. C. Hancox, "Inhibition of the current of heterologously expressed HERG potassium channels by flecainide and comparison with quinidine, propafenone and lignocaine," Br. J. Pharmacol., vol. 136, no. 5, pp. 717-729, Jul. 2002.

[10] M. J. McPate, R. S. Duncan, J. C. Hancox, and H. J. Witchel, "Pharmacology of the short QT syndrome N588K-hERG K+ channel mutation: differential impact on selected class I and class III antiarrhythmic drugs," $B r . J$. Pharmacol., vol. 155, no. 6, pp. 957-966, Nov. 2008.

[11] A. R. Harmer et al., "Optimisation and validation of a medium-throughput electrophysiology-based hNav1.5 assay using IonWorks ${ }^{\mathrm{TM}}, " J$. Pharmacol. Toxicol. Methods, vol. 57, no. 1, pp. 30-41, Jan. 2008.

[12] J. C. Hancox and J. S. Mitcheson, "Inhibition of L-type calcium current by propafenone in single myocytes isolated from the rabbit atrioventricular node," $\mathrm{Br} . \mathrm{J}$. Pharmacol., vol. 121, no. 1, pp. 7-14, May 1997.

[13] G. J. Gross and N. A. Castle, "Propafenone inhibition of human atrial myocyte repolarizing currents," J. Mol. Cell. Cardiol., vol. 30, no. 4, pp. 783-793, Apr. 1998.

[14] I. Amorós et al., "Propafenone blocks human cardiac Kir2.x channels by decreasing the negative electrostatic charge in the cytoplasmic pore," Biochem. Pharmacol., vol. 86, no. 2, pp. 267-278, Jul. 2013.

[15] L. Franqueza, C. Valenzuela, E. Delpón, M. Longobardo, R. Caballero, and J. Tamargo, "Effects of propafenone and 5-hydroxy-propafenone on hKv1.5 channels," $\mathrm{Br}$. J. Pharmacol., vol. 125, no. 5, pp. 969-978, Nov. 1998.

[16] A. Burashnikov, L. Belardinelli, and C. Antzelevitch, "Atrial-selective sodium channel block strategy to suppress atrial fibrillation: Ranolazine versus propafenone," $J$. Pharmacol. Exp. Ther., vol. 340, no. 1, pp. 161-168, Jan. 2012.

[17] D. G. Whittaker, H. Ni, A. E. Harchi, J. C. Hancox, and H. Zhang, "Atrial arrhythmogenicity of KCNJ2 mutations in short QT syndrome: Insights from virtual human atria," PLOS Comput. Biol., vol. 13, no. 6, p. e1005593, Jun. 2017.

[18] D. G. Whittaker, M. A. Colman, H. Ni, J. C. Hancox, and H. Zhang, "Human atrial arrhythmogenesis and sinus bradycardia in KCNQ1-linked short QT syndrome: Insights from computational modelling," Front. Physiol., vol. $9,2018$.

Address for correspondence.

Dominic G Whittaker.

Garstang 7.53,

University of Leeds,

Leeds, UK,

LS2 9JT,

d.whittaker@leeds.ac.uk 